\title{
SCHATTEN CLASS HANKEL OPERATORS ON THE BERGMAN SPACES OF STRONGLY PSEUDOCONVEX DOMAINS
}

\author{
HUIPING LI
}

(Communicated by Eric Bedford)

\begin{abstract}
In this paper, we characterize holomorphic functions $f$ such that the Hankel operators $H_{f}$ are in the Schatten classes on bounded strongly pseudoconvex domains. It is proved that for $p>2 n, H_{\tilde{f}}$ is in the Schatten class $S_{p}$ if and only if $f$ is in the Besov space $B_{p}$; for $p \leq 2 n, H_{\bar{f}}$ is in the Schatten class $S_{p}$ if and only if $f=$ constant .
\end{abstract}

\section{INTRODUCTION}

Let $D$ be a bounded strongly pseudoconvex domain with smooth boundary in $\mathbb{C}^{n}, n \geq 2$. Let $H^{2}(D)$ be the Bergman space consisting of holomorphic $L^{2}$ functions. The Bergman projection $P$ is the orthogonal projection from $L^{2}(D)$ onto $H^{2}(D)$ defined by $P f(z)=\int K(z, w) f(w) d v(w)$. Here $K(z, w)$ is the Bergman kernel of $D$. For $f \in L^{2}(D)$, the Hankel operator $H_{f}$ from $H^{2}(D)$ into $L^{2}(D)$ is defined by $H_{f}(g)=(I-P)(f \cdot g) . \quad H_{f}$ is densely defined. In [12], we have characterized the functions $f \in H^{2}(D)$ such that $H_{\vec{f}}$ are bounded and compact by functions in the Bloch space and the little Bloch space, respectively. Recently, Arazy, Fisher, Janson, and Peetre [2], Wallsten [16], and Zhu [17] characterized the functions $f \in H^{2}(D)$ such that $H_{\vec{f}} \in S_{p}$ on the unit ball in $\mathbb{C}^{n}$. In their theorems, there is an interesting cutoff property, i.e., if $p>2 n$, then $H_{f} \in S_{p}$ if and only if $f$ is in the holomorphic Besov space $B_{p}$; if $p \leq 2 n$, then $H_{f} \in S_{p}$ if and only if $f$ is a constant. In this paper, we extend those results to bounded strongly pseudoconvex domains with smooth boundaries in $\mathbb{C}^{n}, n>1$. Since there is no nontrivial holomorphic automorphism for general strongly pseudoconvex domains in $\mathbb{C}^{n}$, the methods used here are new and different from those used in [2,16, 17]. The main tools used here are the integral representations of solutions to the $\bar{\partial}$-equation and the integral criterion for extending functions on the boundary $\partial D$ holomorphically into $D$. To state our main results, we need some definitions and notations.

Let $k_{z}(w)=K(w, z) / K(z, z)^{1 / 2}$. Define the Berezin transform of $f \in L^{2}$

Received by the editors March 30, 1992.

1991 Mathematics Subject Classification. Primary 32H10, 47B35; Secondary 32F20.

Research partially supported by a grant of the National Science Foundation. 
as [4]

$$
\tilde{f}(z)=\int f(w)\left|k_{z}(w)\right|^{2} d v(w) .
$$

If $f \in L^{2}(D)$, we write $M O(f, z)=\left(|f|^{2}\right)^{\sim}(z)-|\tilde{f}(z)|^{2}$. Then $M O(f, z)$ is a continuous function on $D$. It is easy to check that for holomorphic functions $f, \widetilde{f}(z)=f(z)$ and

$$
M O(f, z)=\int_{D}|f(z)-f(w)|^{2}\left|k_{z}(w)\right|^{2} d w .
$$

For a $(p, q)$-form $F(z)=\sum F_{I, J}(z) d z_{I} \wedge d \bar{z}_{J}$, where $F_{I, J}$ are complexvalued functions on $D, d z_{I}=d z_{i_{1}} \wedge d z_{i_{2}} \wedge \cdots \wedge d z_{i_{p}}$, and $d \bar{z}_{J}=d \bar{z}_{j_{1}} \wedge d \bar{z}_{j_{2}} \wedge$ $\cdots \wedge d \bar{z}_{j_{q}}$, let

$$
\bar{\partial} F=\sum_{1}^{n} \sum \partial F_{I, J} / \partial \bar{z}_{i} d \bar{z}_{i} \wedge d z_{I} \wedge d \bar{z}_{J}
$$

where $\partial / \partial z_{i}=1 / 2 \cdot\left(\partial / \partial x_{i}-\sqrt{-1} \cdot \partial / \partial y_{i}\right), \partial / \partial \bar{z}_{i}=1 / 2 \cdot\left(\partial / \partial x_{i}+\sqrt{-1} \cdot \partial / \partial y_{i}\right)$. We denote

$$
|F(z)|=\sum\left|F_{I, J}(z)\right| \text {. }
$$

Let $\rho(z) \in C^{\infty}(\bar{D})$ be a strictly plurisubharmonic defining function of $D$ such that $D=\left\{z \in \mathbb{C}^{n}: \rho(z)<0\right\}$ and $\nabla \rho(z) \neq 0$ for $z \in \partial D$, where $\nabla \rho$ is the gradient of $\rho$.

The complex tangential space at a boundary point $p \in \partial D$ is the set

$$
T_{p}^{\mathbb{C}}(D)=\left\{\xi \in \mathbb{C}^{n}: \sum_{1}^{n} \partial \rho(p) / \partial z_{i} \cdot \xi_{i}=0\right\} .
$$

For $2 n<p<\infty$, we define the Besov space $B_{p}$ of holomorphic functions as

$$
B_{p}=\left\{f \in H^{2}(D):|\bar{\partial} \bar{f}| \cdot|\rho|^{1-(n+1) / p} \in L^{p}(D)\right\} .
$$

By the work of Grellier [10], it follows that if $f \in B_{p}$, then $|\bar{\partial} \bar{f} \wedge \bar{\partial} \rho| \cdot$ $|\rho|^{1 / 2-(n+1) / p} \in L^{p}(D)$. We will use this result without further comment.

Throughout this paper, constants are denoted by the letter $C$, and they may change from line to line.

Theorem A. Let $D$ be a connected and bounded strongly pseudoconvex domain with smooth boundary in $\mathbb{C}^{n}, n \geq 2$. Let $f \in H^{2}(D)$.

(1) For $p>2 n$, the following statements are equivalent:

(a) $H_{\tilde{f}} \in S_{p}$;

(b) $f \in B_{p}$

(c) $M O(f, z)^{1 / 2} \cdot K(z, z)^{1 / p} \in L^{p}(D)$.

(2) For $p \leq 2 n, H_{f} \in S_{p}$ if and only if $f$ is a constant.

In $\S 2$, we construct a special weighted integral operator $T$ which solves the $\bar{\partial}$-equation, and prove that for $p>2 n$, if $\psi \in C^{2}(D) \cap L^{2}(D)$ satisfies $\mid \bar{\partial} \psi \wedge$ $\left.\bar{\partial} \rho|\cdot| \rho\right|^{1 / 2-(n+1) / p}+|\bar{\partial} \psi| \cdot|\rho|^{1-(n+1) / p} \in L^{p}(D)$, then the operator $T_{\psi}(h)=$ $T(h \cdot \bar{\partial} \psi)$ is in the Schatten class $S_{p}$ as an operator from $H^{2}(D)$ into $L^{2}(D)$, and consequently, $H_{\psi}=(I-P) T_{\psi} \in S_{p}$. In $\S 3$, we prove that for $f \in H^{2}(D)$, if $H_{\tilde{f}} \in S_{p}$ with $p>2 n$, then $f \in B_{p}$; if $H_{\tilde{f}} \in S_{p}$ with $p \leq 2 n$, then $f$ 
has nontangential limit $f_{b} \in L^{2}(\partial D)$ and $\bar{f}_{b}$ satisfies the weakly tangential Cauchy-Riemann equation [6] on $\partial D$ which implies that $f$ is a constant.

After this paper was written, Marco M. Peloso informed me that he obtained similar results independently.

\section{SUFFICIENCY}

In this section, we prove $(c) \Rightarrow(b) \Rightarrow(a)$ in Theorem $A$.

From now on, we will fix a bounded strongly pseudoconvex domain $D$ with smooth boundary and let $\rho(z) \in C^{\infty}(\bar{D})$ be a strictly plurisubharmonic defining function of $D$. To simplify notation, we shall write $\rho_{i}(z)=\partial \rho(z) / \partial z_{i}$, $\rho_{i j}(z)=\partial^{2} \rho(z) / \partial z_{i} \partial z_{j}$, where $1 \leq i, j \leq n$. Let $F_{1}(z, w)$ denote the Levi polynomial

$$
F_{1}(z, w)=\sum_{i=1}^{n} \rho_{i}(w)\left(w_{i}-z_{i}\right)-\frac{1}{2} \cdot \sum_{i, j=1}^{n} \rho_{i j}(w)\left(w_{i}-z_{i}\right)\left(w_{j}-z_{j}\right) .
$$

It is well known [13] that there exist constants $\delta$ and $C_{1}$ such that for $z, w \in \bar{D}$ with $|z-w| \leq \delta, \operatorname{Re}\left(F_{1}(z, w)-\rho(w)\right) \geq C_{1} \cdot\left(-\rho(z)-\rho(w)+|z-w|^{2}\right)$.

Before going on, we collect some facts which will be needed later.

Lemma $2.1[3,13]$. Let $\rho$ and $\delta$ be the same as above. There exist functions $h_{i}(z, w), 1 \leq i \leq n$, and $\Psi(z, w)$ in $C^{\infty}(\bar{D} \times \bar{D})$ such that

(1) For each fixed $w \in \bar{D}, h_{i}(z, w)$ and $\Psi(z, w)$ are holomorphic in $z \in \bar{D}$.

(2) $\Psi(w, w)=-\rho(w)$ and there is a nonvanishing smooth function $g(z, w)$ in $\bar{D} \times \bar{D}$ such that if $|z-w| \leq \delta / 2$, then $\Psi(z, w)=g(z, w) \cdot$ $\left(F_{1}(z, w)-\rho(w)\right)$; if $|z-w| \geq \delta / 2$, then $|\Psi(z, w)| \geq 1 / C$.

(3) $\Psi(z, w)=\sum_{i=1}^{n} h_{i}(z, w)\left(w_{i}-z_{i}\right)-\rho(w)$.

(4) $h_{i}(w, w)=\rho_{i}(w)-\rho(w) \cdot g_{i}(w)$, where $g_{i}(w)=\partial g(z, w) /\left.\partial w_{i}\right|_{z=w}$.

Write

$$
G(z, w)=\sum \rho_{i}(z)\left(z_{i}-w_{i}\right)-\frac{1}{2} \cdot \sum \rho_{i j}(z)\left(z_{i}-w_{i}\right)\left(z_{j}-w_{j}\right) .
$$

Lemma 2.2 [13]. Let $\rho$ and $G$ be the same as above. There exist constants $\delta$ and $c$ such that for any $z \in D$ with $|\rho(z)| \leq \delta$, in the ball $B(z, \delta)$ we can perform a smooth change of variables $\tau=\tau(w)$ with the properties

(1) $\tau_{1}(w)=G(z, w)$;

(2) $|z-w| / c \leq|\tau(w)| \leq c \cdot|z-w|$ for $w \in B(z, \delta)$;

(3) $1 / c \leq|\partial \tau / \partial w| \leq c$ for $w \in B(z, \delta)$, where $\partial \tau / \partial w$ denotes the Jacobian of $\tau$.

For any $w \in D$ with $|\rho(w)| \leq \delta$, in the ball $B(w, \delta)$ we can perform a smooth change of variables $\lambda=\lambda(z)$ with $\lambda_{1}(z)=G(z, w)$ such that (2) and (3) hold for $\lambda(z)$.

Remark. In standard texts [1, 13], the coordinates $\tau=\tau(w)$ with $\operatorname{Re} \tau_{1}=$ $\rho(w)-\rho(z)$ (or $\left.\operatorname{Re} \tau_{1}=\rho(w)\right)$ and $\operatorname{Im} \tau_{1}=\operatorname{Im} G(z, w)$ are used. Since we are going to estimate some special integrals, we need the coordinate system in the lemma.

Choose a smooth function $\chi$ on $\mathbb{C}^{n} \times \mathbb{C}^{n}$ such that $0 \leq \chi \leq 1$ and

$$
\chi(z, w)= \begin{cases}1 & \text { if }|z-w| \leq \delta / 2 \\ 0 & \text { if }|z-w| \geq \delta\end{cases}
$$


Write

$$
\begin{gathered}
G_{1}(z, w)=\chi \cdot G(z, w)+(1-\chi)|z-w|^{2}, \\
\|z-w\|_{A}^{2}=\left|G_{1}(z, w)\right|^{2}+|\rho(z)| \cdot|z-w|^{2}, \\
s_{i}(z, w)=\overline{G_{1}(z, w)} \cdot\left[\chi \cdot\left(\rho_{i}(z)-\frac{1}{2} \cdot \sum_{j=1}^{n} \rho_{i j}(z)\left(z_{j}-w_{j}\right)\right)\right. \\
\left.+(1-\chi) \cdot\left(\overline{z_{i}-w_{i}}\right)\right]+|\rho(z)| \cdot \overline{\left(z_{i}-w_{i}\right)} .
\end{gathered}
$$

It is obvious that $\|z-w\|_{A}^{2}=\sum s_{i}(z, w) \cdot\left(z_{i}-w_{i}\right),\left|s_{i}\right| \leq C \cdot|z-w|$ uniformly for $z, w \in \bar{D}$, and for $z$ in any compact subset $\Omega \subset D,\|z-w\|_{A}^{2} \geq C^{\prime} \cdot|z-w|^{2}$ uniformly for $w \in \bar{D}$, where $C^{\prime}$ may depend on the compact subset $\Omega$. Thus, the $s_{i}$ satisfy condition (1) in [5].

Lemma 2.3. (1) If $|z-w|<\delta / 2$, then $\|z-w\|_{A}^{2} \geq 1 / C \cdot\left(|G(z, w)|^{2}+|\rho(w)| \cdot\right.$ $\left.|z-w|^{2}\right)$.

(2) If $|z-w| \geq \delta / 2$, then $\|z-w\|_{A}^{2} \geq 1 / C \cdot|\rho(w)|$.

Proof. If $|\rho(z)| \geq 1 / 2 \cdot|\rho(w)|$ or $|z-w|>\delta$, it is obvious that the results hold. If $|\rho(z)|<1 / 2 \cdot|\rho(w)|$ and $|z-w| \leq \delta$, note that $\rho(w)$ is a $C^{\infty}$ strictly plurisubharmonic function in a neighborhood of $\bar{D}$, by the Taylor expansion of $\rho(w)$ at $z$, then

$$
\begin{aligned}
\operatorname{Re} G_{1}(z, w) & \geq \chi / 2 \cdot\left(-\rho(w)+\rho(z)+C_{1} \cdot|z-w|^{2}\right)+(1-\chi) \cdot|z-w|^{2} \\
& \geq \chi / 2 \cdot\left(-1 / 2 \cdot \rho(w)+C_{1} \cdot|z-w|^{2}\right)+(1-\chi) \cdot|z-w|^{2}
\end{aligned}
$$

Thus, for $|z-w|<\delta / 2$,

$$
\begin{aligned}
\left|G_{1}\right|^{2} & =|G|^{2} \geq 1 / 2 \cdot|G|^{2}+C_{2} \cdot\left(|\rho(w)|^{2}+|z-w|^{4}\right) \\
& \geq 1 / 2 \cdot|G|^{2}+2 \cdot C_{2} \cdot|\rho(w)||z-w|^{2}
\end{aligned}
$$

This finishes the proof of assertion (1).

For $\delta / 2 \leq|z-w| \leq \delta$, by $(*)$ we have $\left|G_{1}\right|^{2} \geq\left[\min \left\{C_{1} / 2,1\right\}\right]^{2}$. $|z-w|^{4} \geq C_{2} \cdot \delta^{4}$. Note that $\rho(w) \in C^{\infty}(\bar{D})$; then $1 /|\rho(w)|>C_{3}$. Therefore, $\left|G_{1}\right|^{2} \geq C_{3} C_{2} \delta^{4}|\rho(w)|$. Q.E.D.

Following Berndtsson and Andersson [5], we define

$$
\begin{aligned}
& s(z, w)=\sum_{1}^{n} s_{i}(z, w) d w_{i}, \quad h(z, w)=\sum_{1}^{n} h_{i}(z, w) d w_{i}, \\
& \mu(z, w)=\bar{\partial}_{w} h(z, w) / \rho(w)-\bar{\partial} \rho(w) \wedge h(z, w) / \rho(w)^{2} \\
& L(z, w)=C_{n} \cdot \sum_{0}^{n-1} \gamma_{k} \cdot[-\rho(w) / \Psi(z, w)]^{k+n+1} \\
& \cdot s \wedge \mu^{k} \wedge\left(\bar{\partial}_{w} s\right)^{n-k-1} /\|z-w\|_{A}^{2(n-k)}
\end{aligned}
$$

where $\gamma_{k}$ and $C_{n}$ are some constants [5]. 
It is easy to check that for $|z-w|<\delta / 2$,

$$
\begin{aligned}
s(z, w)=\overline{G(z, w)} \cdot\left[\sum_{1}^{n} \rho_{i}(z) d w_{i}+\beta_{1}(z, w)\right]+|\rho(z)| \cdot \sum_{1}^{n}\left(\bar{z}_{i}-\bar{w}_{i}\right) d w_{i}, \\
\bar{\partial}_{w} s(z, w)=\left[-\sum_{1}^{n} \overline{\rho_{i}(z)} d \bar{w}_{i}+\overline{\beta_{2}(z, w)}\right] \\
\wedge\left[\sum_{1}^{n} \rho_{i}(z) d w_{i}+\beta_{1}(z, w)\right]+\rho(z) \sum_{1}^{n} d \bar{w}_{i} \wedge d w_{i},
\end{aligned}
$$

where $\beta_{k}, k=1,2$, are $(1,0)$-forms with $\left|\beta_{i}(z, w)\right| \leq C \cdot|z-w|$.

Write $\tilde{s}(z, w)=\sum_{1}^{n} \rho_{i}(z) d w_{i}+\beta_{1}(z, w)$. By direct computation we have

$$
\mu^{k}=\left[\rho(w)\left(\bar{\partial}_{w} h\right)^{k}-(k-1) \bar{\partial} \rho(w) \wedge h \wedge\left(\bar{\partial}_{w} h\right)^{k-1}\right] / \rho(w)^{k+1},
$$

and for $|z-w|<\delta / 2$,

$$
\begin{aligned}
\left(\bar{\partial}_{w} s\right)^{m}= & {\left[\rho(z) \sum d \bar{w}_{i} \wedge d w_{i}+(m-1) \bar{\partial}_{w} \overline{G(z, w)} \wedge \tilde{s}(z, w)\right] } \\
& \wedge\left(\rho(z) \sum d \bar{w}_{i} \wedge d w_{i}\right)^{m-1} .
\end{aligned}
$$

Lemma 2.4 [5, pp. 103-104]. If $u$ is a $\bar{\partial}$-closed $(0,1)$-form with coefficients in $C^{1}(\bar{D})$, then

$$
v(z)=T(u)(z)=\int_{D} u(w) \wedge L(z, w)
$$

is a solution to the equation $\bar{\partial} v=u$.

Remark 1 . In [5], the theorem was proved for strictly convex domains by letting

$$
s_{i}=\left[\sum_{k=1}^{k} \overline{\rho_{k}(z)}\left(\bar{z}_{k}-\bar{w}_{k}\right)\right] \cdot \rho_{i}(z)+|\rho(z)| \cdot\left(\bar{z}_{i}-\bar{w}_{i}\right),
$$

and $h_{i}=\rho_{i}(w)$, where $1 \leq i \leq n$. As indicated in [5, p. 104], an application of the same arguments yields the results here.

Remark 2. By a standard argument (see [13, p. 297]), it follows that Lemma 2.4 holds for the $\bar{\partial}$-closed $(0,1)$-forms $u$ with coefficients in $C^{1}(D) \cap L^{1}(D)$.

The next lemma is crucial to our analysis. It seems to me that the standard integral representations and estimates $[1,13]$ do not work in our case; the following estimates should have their own interest. For each $a>0$, we shall write $D_{a}=\{z \in \bar{D}:|\rho(z)|<a\}$.

Lemma 2.5. If $f \in B_{p}$ with $p>2 n$, then for $1 / q+1 / p=1$,

$$
\begin{aligned}
& \int_{D}\left(\int_{D}|\bar{\partial} \bar{f} \wedge L(z, w)|^{q} d w\right)^{p / q} d z<\infty \\
& \int_{D}\left(\int_{D}|\bar{\partial} \bar{f} \wedge L(z, w)|^{q} d z\right)^{p / q} d w<\infty .
\end{aligned}
$$

Proof. By Lemmas 2.1 and 2.3, it is easy to check that for $|z-w| \geq \delta / 2$,

$$
|\bar{\partial} \bar{f} \wedge L| \leq C \cdot|\rho \cdot \overline{\partial f}|
$$


Note that for $|z-w|<\delta / 2, \partial_{w} G_{1}(z, w)=\partial_{w} G(z, w)=-\partial \rho(w)+$ $e_{1}(z, w), \tilde{s}(z, w)=\sum \rho_{i}(z) d w_{i}+\beta_{1}(z, w)=\partial \rho(w)+e_{2}(z, w)$, and $h(z, w)$ $=\partial \rho(w)+e_{3}(z, w)$, where $e_{i}(i=1,2,3)$ are $(1,0)$-forms with $\left|e_{k}(z, w)\right| \leq$ $C \cdot|z-w|$ for $k=1,2$, and $\left|e_{3}\right| \leq C \cdot(|z-w|+|\rho(w)|)$. It follows that

$$
\begin{gathered}
\left|\bar{\partial} \bar{f}(w) \wedge \bar{\partial}_{w} \overline{G_{1}(z, w)}\right| \leq|\bar{\partial} \bar{f}(w) \wedge \bar{\partial} \rho(w)|+C \cdot|z-w||\overline{\partial f}| \\
|\tilde{s}(z, w) \wedge h(z, w)| \leq C \cdot[|z-w|+|\rho(w)|] \\
\left|\overline{\partial f}(w) \wedge \bar{\partial}_{w} \bar{G}_{1} \wedge \tilde{s}(z, w) \wedge \bar{\partial} \rho(w) \wedge h(z, w)\right| \\
\leq C \cdot|\bar{\partial} \bar{f}(w) \wedge \bar{\partial} \rho(w)||z-w| \cdot(|z-w|+|\rho(w)|)
\end{gathered}
$$

Recall that for $|z-w|<\delta / 2$,

$$
\begin{gathered}
|G(z, w)|^{2}+|\rho(w)| \cdot|z-w|^{2} \leq C \cdot\|z-w\|_{A}^{2} \\
\left|G_{1}(z, w)\right|=|G(z, w)| \leq C \cdot|\Psi(z, w)| \\
|\rho(z)|+|\rho(w)|+|z-w|^{2}+|\operatorname{Im} \Psi(z, w)| \leq C \cdot|\Psi(z, w)| .
\end{gathered}
$$

By the equations given before Lemma 2.4, a straightforward computation yields that

$$
|\overline{\partial f} \wedge L| \leq C \cdot\left(E_{0}+F_{0}+\sum_{1}^{n-1} E_{k}+1\right) \cdot Q
$$

Here

$$
\begin{aligned}
E_{0}= & |\rho(z)|^{n-1}|\rho(w)|^{n+1 / 2+(n+1) / p}|z-w| /\left(\|z-w\|_{A}^{2 n} \cdot|\Psi(z, w)|^{n+1}\right), \\
F_{0}= & \frac{|\rho(z)|^{n-1} \cdot|\rho(w)|^{n}}{\|z-w\|_{A}^{2 n} \cdot|\Psi(z, w)|^{n+1}} \cdot|\rho(w)|^{(n+1) / p} \\
& \cdot\left(|G(z, w)|+|\rho(z)||z-w|+|z-w|^{2}\right) \\
E_{k}= & |\rho(z)|^{n-k-1} \cdot|\rho(w)|^{n-1 / 2+(n+1) / p} /\left(\|z-w\|_{A}^{2(n-k)}|\Psi(z, w)|^{n+k-1 / 2}\right),
\end{aligned}
$$

where $1 \leq k<n-1$,

$$
E_{n-1}=|\rho(w)|^{(n+1) / p} /\left(|z-w| \cdot|\Psi(z, w)|^{n+1 / 2}\right),
$$

and

$$
Q(w)=\left(|\bar{\partial} \rho(w) \wedge \bar{\partial} \bar{f}| \cdot|\rho(w)|^{1 / 2}+|\bar{\partial} \bar{f}(w)| \cdot|\rho(w)|\right) /|\rho(w)|^{(n+1) / p} .
$$

To prove (2.3) and (2.4), we show that for $q-1<\varepsilon<1$ and $0 \leq i \leq n-1$,

$$
\begin{gathered}
\int_{D} E_{i}(z, w)^{q} \cdot|\rho(w)|^{-\varepsilon} d w \leq C \cdot|\rho(z)|^{-\varepsilon} \\
\int_{D} E_{i}(z, w)^{q} \cdot|\rho(z)|^{-\varepsilon} d z \leq C \cdot|\rho(w)|^{-\varepsilon} \\
\int_{D} E_{i}(z, w)^{q} d z \leq C
\end{gathered}
$$

where $C$ is a constant independent of $z, w \in D$; the same estimates hold for $F_{0}$.

By Lemmas 2.1 and 2.3, if $|z-w| \geq \delta / 2$, then $E_{i}$ and $F_{0}$ are bounded by a constant $C$. If $|\rho(z)| \geq \delta$ and $|z-w|<\delta / 2$, then $E_{i}^{q} \cdot|\rho(w)|^{-\varepsilon}$ and 
$F_{0}^{q} \cdot|\rho(w)|^{-\varepsilon}$ are bounded above by $C /|z-w|^{(2 n-1) q}$, and similar results hold when $|\rho(w)| \geq \delta$. Note that $(2 n-1) q<2 n$ when $p>2 n$; it is easy to prove $(2.5)-(2.7)$ for those cases. Thus, it suffices to prove (2.5)-(2.7) for $z$, $w \in D_{\delta}$ and integrals over $|z-w|<\delta / 2$. Since the proofs of those estimates for $E_{0}$ and $F_{0}$ are similar, we shall only prove $(2.5)-(2.7)$ for $E_{0}(z, w)$ and $E_{k}, 1 \leq k \leq n-1$, respectively.

For $z \in D_{\delta}$ and $w \in B=B(z, \delta / 2)$, note that

$$
|\Psi(z, w)| \geq C \cdot\left(|\rho(z)|+|\rho(w)|+|z-w|^{2}\right) ;
$$

then

$$
\begin{aligned}
& I_{0}= \int_{B} E_{0}(z, w)^{q}|\rho(w)|^{-\varepsilon} d w \\
& \leq C /|\rho(z)|^{q} \cdot \int_{B}\left(|z-w|^{2}+|G|^{2} /|\rho(z)|\right)^{-(n-1 / 2) q} \\
& \cdot\left(|\rho(z)|+|z-w|^{2}\right)^{-\varepsilon-q / 2+(n+1) q / p} d w .
\end{aligned}
$$

Using the coordinate system in Lemma 2.2 and writing $\tau^{\prime}=\left(\tau_{2}, \ldots, \tau_{n}\right) \in$ $\mathbb{C}^{n-1}$, one has

$I_{0} \leq C /|\rho(z)|^{q} \cdot \int_{|\tau| \leq 1}\left(\left|\tau^{\prime}\right|^{2}+\left|\tau_{1}\right|^{2} /|\rho(z)|\right)^{-(n-1 / 2) q}\left(|\rho(z)|+\left|\tau^{\prime}\right|^{2}\right)^{-\varepsilon-q / 2+(n+1) q / p} d \tau$.

Integrate with respect to $\tau_{1}$ over the unit disc $\left|\tau_{1}\right| \leq 1$ in $\mathbb{C}$, and then let $\tau^{\prime}=\sqrt{|\rho(z)|} \cdot \eta^{\prime} ;$ we have

$$
I_{0} \leq C /|\rho(z)|^{\varepsilon} \cdot \int_{\mathbf{C}^{n-1}}\left|\eta^{\prime}\right|^{-(2 n-1) q+2}\left(1+\left|\eta^{\prime}\right|^{2}\right)^{-\varepsilon-q / 2+(n+1) q / p} d \eta^{\prime} .
$$

Note that for $p>2 n$ and $\varepsilon>q-1,(2 n-1) q-2<2 n-2$ and $(2 n-1) q-$ $2+2 \varepsilon+q-2(n+1) q / p>2 n-2$. It follows that the integral on the right side of $(2.8)$ is finite. Thus, $I_{0}<\infty$.

Next we prove (2.6) for $E_{0}(z, w)$. By Lemmas 2.2 and 2.3, we have

$$
\begin{aligned}
& I_{1}=\int_{B(w, \delta / 2)} E_{0}(z, w)^{q}|\rho(z)|^{-\varepsilon} d z \\
& \leq C \cdot|\rho(w)|^{(n+1) q / p} \int_{B}\left(|z-w|^{2}+|G|^{2} /|\rho(w)|\right)^{-(n-1 / 2) q} \\
& \cdot\left(|\rho(w)|+|z-w|^{2}\right)^{-3 q / 2-\varepsilon} d z \\
& \leq C \cdot|\rho(w)|^{(n+1) q / p} \int_{|\tau| \leq 1}\left(\left|\tau^{\prime}\right|^{2}+\left|\tau_{1}\right|^{2} /|\rho(w)|\right)^{-(n-1 / 2) q} \\
& \cdot\left(|\rho(w)|+\left|\tau^{\prime}\right|^{2}\right)^{-3 q / 2-\varepsilon} d \tau .
\end{aligned}
$$

By the same arguments as those used in the proof of $I_{0}<\infty$, one can prove that $I_{1}<\infty$. This completes the proof of (2.6) for $E_{0}(z, w)$. Similarly, we can prove (2.7) for $E_{0}$. 
Now we prove $(2.5)-(2.7)$ for $E_{k}, 1 \leq k<n-1$. By Lemmas 2.1-2.3, it follows that

$$
\begin{aligned}
I_{2} & =\int_{B(z, \delta / 2)} E_{k}(z, w)^{q}|\rho(w)|^{-\varepsilon} d w \\
& \leq C \cdot \int_{|\tau| \leq 1}|\rho(z)|^{(n-k-1) q}\left(|\rho(z)|\left|\tau^{\prime}\right|^{2}+\left|\tau_{1}\right|^{2}\right)^{-n q+k q} \\
& \cdot\left(|\rho(z)|+\left|\tau^{\prime}\right|^{2}\right)^{-k q+(n+1) q / p-\varepsilon} d \tau .
\end{aligned}
$$

Let $\tau_{1}=|\rho(z)| \cdot \eta_{1}$ and $\tau^{\prime}=\sqrt{|\rho(z)|} \cdot \eta^{\prime}$. Then

$$
I_{2} \leq C /|\rho(z)|^{\varepsilon} \cdot \int_{\mathbb{C}^{n}}\left(|\eta|^{2}\right)^{-n q+k q} \cdot\left(1+\left|\eta^{\prime}\right|^{2}\right)^{-k q+(n+1) q / p-\varepsilon} d \eta .
$$

Note that $-1>-n q+k q>-n$ and $-n q+k q-k q+(n+1) q / p-\varepsilon<-n$. It is obvious that the integral on the right side of $(2.9)$ is finite. Thus, we get (2.5) for $E_{k}$.

Again, by Lemma 2.3, $\|z-w\|_{A}^{2} \geq 1 / C \cdot\left(|G(z, w)|^{2}+|\rho(w)| \cdot|z-w|^{2}\right)$. Then

$$
\begin{aligned}
E_{k} \leq & C|\rho(z)|^{\varepsilon / q}|\rho(w)|^{n-1 / 2+(n+1) / p} \\
& \cdot\left(|G|^{2}+|\rho(w)||z-w|^{2}\right)^{k-n}|\Psi(z, w)|^{-2 k-1 / 2-\varepsilon / q} .
\end{aligned}
$$

By repeating the procedure above, one can prove (2.6) and (2.7) for $E_{k}, 1 \leq$ $k<n-1$.

It remains to prove $(2.5)-(2.7)$ for $E_{n-1}$. In this case, the results can be proved by using the coordinate systems and methods given in [13, pp. 299-300] except for obvious modifications. We omit the details here.

Note that $f \in B_{p}$ implies that $Q \in L^{p}(D)$. An application of the arguments used in the proof of Lemma 5 in [18] to $(* *),(2.5)$, and (2.6) yields (2.3); by $(* *)$ and $(2.7)$ it follows that $(2.4)$ holds. This finishes the proof of our lemma. Q.E.D.

Theorem 2.6. For $f \in B_{p}$ with $p>2 n$, let $T_{0}(g)=T(g \cdot \bar{\partial} \bar{f})$ for $g \in H^{2}(D)$. Then $T_{0}$ is in the Schatten class $S_{p}$ as an operator from $H^{2}(D)$ into $L^{2}(D)$.

Proof. By Lemma 2.5 and Russo's theorem [14], it follows that $T_{0}(g)=$ $T(\bar{\partial}(\bar{f} g))$ is an operator in $S_{p}$. Q.E.D.

Theorem 2.7. If $f \in B_{p}$ with $p>2 n$, then $H_{\tilde{f}} \in S_{p}$.

Proof. For $g \in H^{\infty}(D)$, the space of bounded holomorphic functions in $D$, it is easy to check that $f \in B_{p}$ implies that $|\bar{\partial}(\bar{f} \cdot g)| \in L^{1}(D)$. By Lemma 2.4 and Theorem 2.6, $u=T_{0}(g)=T(\bar{\partial}(\bar{f} g))$ is a solution to the equation $\bar{\partial} u=\bar{\partial}(\bar{f} g)=g \cdot \bar{\partial} \bar{f}$ and $u \in L^{2}(D)$. Obviously, $\bar{f} \cdot g \in L^{2}(D)$ is a solution to the same equation. By the uniqueness of the solution orthogonal to $H^{2}(D)$, it follows that $H_{\bar{f}} g=(I-P)(\bar{f} g)=(I-P)(u)=(I-P)\left(T_{0}(g)\right)$. Since $H^{\infty}(D)$ is dense in $H^{2}(D)$ [13], Theorem 2.6 implies that $H_{\bar{f}} \in S_{p}$. Q.E.D.

Remark. In the proofs of Lemma 2.5 and Theorem 2.7 , we have actually proved that if $\psi \in C^{2}(D) \cap L^{2}(D)$ satisfies $\left(|\bar{\partial} \psi \wedge \bar{\partial} \rho| \cdot|\rho|^{1 / 2}+|\rho \cdot \bar{\partial} \psi|\right) /|\rho|^{(n+1) / p} \in$ $L^{p}(D)$, then the Hankel operator $H_{\psi} \in S_{p}$. 
Theorem 2.8. For $f \in H^{2}(D)$, if $M O(f, z)^{1 / 2} K(z, z)^{1 / p} \in L^{p}(D)$, then $f \in B_{p}$ when $p>2 n$.

Proof. Note that if $f \in H^{2}(D)$, then $\tilde{f}(z)=f(z)$. By Theorem $\mathrm{F}$ in [4], it follows that for any $\xi \in \mathbb{C}^{n},\left|f_{*}(z) \xi\right| \leq C \cdot M O(f, z)^{1 / 2} \cdot S(z, \xi)$, where $S(z, \xi)$ is the infinitesimal form of the Bergman metric (for the definition of $S(z, \xi)$ see [13]), and $f_{*}(z) \xi=\sum \partial f(z) / \partial z_{i} \cdot \xi_{i}$. By the boundary behaviors of the Bergman metric [9], it follows that for $z$ close to $\partial D,\left|f_{*}(z) \xi\right| \leq$ $C \cdot M O(f, z)^{1 / 2} \cdot\left[\left|\xi_{T}\right| /|\rho(z)|^{1 / 2}+\left|\xi_{N}\right| /|\rho(z)|\right]$, where $\xi_{T}$ and $\xi_{N}$ are the components of $\xi$ in the complex tangent directions and complex normal direction at $\pi(z)$, and $\pi(z)$ is the normal projection of $z$ on $\partial D$. Thus, $|\rho \cdot \partial f| \leq$ $C \cdot M O(f, z)^{1 / 2}$. Note that [8] $K(z, z) / C \leq|\rho(z)|^{-(n+1)} \leq C \cdot K(z, z)$. Thus, $M O(f, z)^{1 / 2} K(z, z)^{1 / p} \in L^{p}(D)$ implies that $|\rho \cdot \bar{\partial} \bar{f}| \cdot|\rho|^{-(n+1) / p} \in L^{p}(D)$. Therefore, when $p>2 n, f \in B_{p}$. Q.E.D.

\section{NeCESSITY}

In this section, we prove $(\mathrm{a}) \Rightarrow(\mathrm{c})$ and $(2)$ of Theorem $\mathrm{A}$.

Theorem 3.1. For $f \in H^{2}(D)$ and $p \geq 2$, if $H_{\bar{f}} \in S_{p}$, then

$$
M O(f, z)^{1 / 2} K(z, z)^{1 / p} \in L^{p}(D) .
$$

Proof. It is easy to check that $H_{\bar{f}} k_{z}(w)=(\overline{f(w)}-\overline{f(z)}) \cdot k_{z}(w)$ and $\left\|H_{\bar{f}} k_{z}\right\|^{2}=$ $M O(f, z)$. Note that for $z \in D, k_{z}(\cdot)$ are unit vectors in $H^{2}(D)$. By the same arguments as those given in $[2,17]$, the result follows. Q.E.D.

Theorem 3.2. Let $f \in H^{2}(D)$ and $p \geq 2$. If $H_{f} \in S_{p}$, then $|\nabla f| \in L^{\alpha}(D)$ for some $\alpha>1$. Moreover, if $p>2 n$, then $H_{f} \in S_{p}$ implies that $f \in B_{p}$.

Proof. The second assertion follows from Theorem 2.8 and Lemma 3.1. To prove the first result, by Lemma 3.1 and the proof of Theorem 2.8, one has $|\nabla f| \cdot|\rho|^{1-(n+1) / p} \in L^{p}(D)$. Note that $|\rho|^{-\beta} \in L^{1}(D)$ for any $0<\beta<1$. By direct calculation using Hölder's inequality we have $|\nabla f| \in L^{\alpha}(D)$ for some $\alpha>1$. Q.E.D.

Theorem 3.3. If $H_{\tilde{f}} \in S_{p}, p \leq 2 n$, then $f=$ constant .

Proof. Since $S_{\alpha} \subset S_{\beta}$ if $\alpha<\beta$, it suffices to prove the result for $p=2 n$. If $H_{\tilde{f}} \in S_{2 n}$, then $H_{\tilde{f}}$ is compact. By Theorem E in [12], $f \in B_{0}(D)=\{g \in$ $H^{2}(D):|\nabla g \cdot \rho| \rightarrow 0$ as $\left.z \rightarrow \partial D\right\}$. Note that [7] $B_{0}(D) \subset P\left(L^{\infty}(D)\right)$ and the Bergman projection is bounded [18] on $L^{r}(D)$ for $1<r<\infty$. Thus $f \in H^{r}(D)$ for any $1<r<\infty$. By Lemma 3.2, $f \in S_{2 n}$ implies that $|\nabla f| \in L^{\alpha}(D)$ for some $\alpha>1$. By direct computation using Stokes theorem one can easily check that $f$ is in the Hardy space $h^{2}(D)$. It is well known [15] that $f \in h^{2}$ implies that $f$ has nontangential boundary values $f_{b} \in L^{2}(\partial D)$. We claim that for any smooth $(n, n-2)$-form $\gamma$ on $\bar{D}, \int_{\partial D} \bar{f}_{b} \cdot \bar{\partial} \gamma=0$. In fact, by the definition of $f_{b}$ and the Stokes Theorem, we have

$$
\int_{\partial D} \bar{f}_{b} \bar{\partial} \gamma=\lim _{\varepsilon \rightarrow 0} \int_{\partial D_{\varepsilon}} \bar{f} \bar{\partial} \gamma=\lim _{\varepsilon \rightarrow 0} \int_{\partial D_{\varepsilon}}-\bar{\partial} \bar{f} \wedge \gamma,
$$

where $D_{\varepsilon}=\{z \in D: \rho(z)=-\varepsilon\}$. Note that $[6,11] \bar{\partial} \bar{f}=\bar{\partial}_{b} \bar{f}+\bar{\partial}_{\nu} \bar{f}$, where $\bar{\partial}_{b} \bar{f}$ and $\bar{\partial}_{\nu} \bar{f}$ are complex tangential and complex normal components of $\bar{\partial} \bar{f}$ 
on $\partial D_{\varepsilon}$, respectively. Since $\gamma$ is a smooth $(n, n-2)$-form, it follows that [6, p. 617] $\int_{\partial D_{\varepsilon}} \bar{\partial} \bar{f} \wedge \gamma=\int_{\partial D_{\varepsilon}} \bar{\partial}_{b} \bar{f} \wedge \gamma$. Thus

$$
\int_{\partial D} \bar{f}_{b} \bar{\partial} \gamma=\lim _{\varepsilon \rightarrow 0} \int_{\partial D_{\varepsilon}}-\bar{\partial}_{b} \bar{f} \wedge \gamma
$$

Note that the coefficients of $\bar{\partial}_{b} \bar{f}(z)$ are of the forms $\sum_{1}^{n} \overline{\partial f / \partial z_{i}} \cdot \bar{\xi}_{i}$, where $\xi$ are vectors in the complex tangential space at $z \in \partial D_{\varepsilon}$ and $|\xi| \leq C$. By Lemma 3.1 and the proof of Theorem 2.8, $H_{\bar{f}} \in S_{2 n}$ implies that $\left|\bar{\partial}_{b} \bar{f}\right||\rho|^{1 / 2}$. $K(z, z)^{1 / 2 n} \in L^{2 n}(D)$. Note that $K(z, z) \approx|\rho(z)|^{-(n+1)}$. We have

$$
\int_{D_{a}}\left|\bar{\partial}_{b} \bar{f}\right|^{2 n} /|\rho(z)| d z=\int_{0}^{a} t^{-1} d t \int_{\partial D_{t}} W(z) \cdot\left|\bar{\partial}_{b} \bar{f}\right|^{2 n} d \sigma<\infty,
$$

where $D_{a}=\{z \in D:|\rho(z)|<a\}, \partial D_{t}=\{z \in D: \rho(z)=-t\}$, and $W>0$ is a smooth function bounded from 0 when $a$ is small.

From (3.2) one can easily obtain that there is a sequence $t_{n} \rightarrow 0$ such that

$$
\lim _{n \rightarrow \infty} \int_{\partial D_{t_{n}}}\left|\bar{\partial}_{b} \bar{f}\right|^{2 n} d \sigma \rightarrow 0
$$

Consequently,

$$
\lim _{n \rightarrow \infty} \int_{\partial D_{t_{n}}}\left|\bar{\partial}_{b} \bar{f}\right| d \sigma=0
$$

An application of (3.3) to (3.1) yields that $\int_{\partial D} \bar{f}_{b} \wedge \bar{\partial} \gamma=0$.

By Theorem 10 in [6, p. 617], it follows that $\bar{f}_{b}$ has a holomorphic extension $f_{1} \in h^{2}(D)$. Note that $f$ is the holomorphic extension of $f_{b}$ in $D$. By the reproducing property of the Poisson-Szegö kernel [15], it follows that both $f$ and $\bar{f}$ are holomorphic in $D$. Consequently, $f \equiv$ constant . Q.E.D.

\section{REFERENCES}

1. L. A. Aizenberg and S. A. Dautov, Differential forms orthogonal to holomorphic functions or forms, and their properties, Transl. Math. Monographs, vol. 56, Amer. Math. Soc. Providence, RI, 1983.

2. J. Arazy, S. Fisher, S. Janson, and J. Peetre, Membership of Hankel operators on the ball in unitary ideals, J. London Math. Soc. 43 (1991), 485-508.

3. F. Beatrous, $L^{p}$ estimates for extensions of holomorphic functions, Michigan Math. J. 32 (1985), 361-380.

4. D. Bekolle, C. A. Berger, L. A. Coburn, and K. Zhu, BMO in the Bergman metric on bounded symmetric domains, J. Funct. Anal. 93 (1990), 310-350.

5. B. Berndtsson and M. Andersson, Henkin-Ramirez formulas with weight factors, Ann. Inst. Fourier (Grenoble) 12 (1982), 91-110.

6. E. M. Chirka and G. M. Khenkin, Boundary properties of holomorphic functions of several complex variables, J. Soviet Math. 5 (1976), 612-687.

7. B. Coupet, Décomposition atomique des espaces de Bergman, Indiana Univ. Math. J. 38 (1989), 917-940.

8. C. Fefferman, The Bergman kernel and biholomorphic mappings of pseudoconvex domains, Invent. Math. 26 (1974), 1-65.

9. I. Graham, Boundary behaviors of the Caratheodory and Kobayashi metrics on strongly pseudoconvex domains in $\mathbb{C}^{n}$ with smooth boundary, Trans. Amer. Math. Soc. 208 (1975), 219-240. 
10. S. Grellier, Comportement des functions holomorphes dans les directions complexes tangentes, C. R. Acad. Sci. Paris Sér. Math. 312 (1991), 267-270.

11. J. J. Kohn and H. Rossi, On the extension of holomorphic functions from the boundary of a complex manifold, Ann. of Math. (2) 81 (1965), 451-472.

12. $\mathrm{H}$. Li, BMO, VMO and Hankel operators on the Bergman spaces of strongly pseudoconvex domains, J. Funct. Anal. 106 (1992), 375-408.

13. R. M. Range, Holomorphic functions and integral representations in several complex variables, Springer-Verlag, Berlin and New York, 1986.

14. B. Russo, On the Hausdorff Young theorem for integral operators, Pacific J. Math. 68 (1977), 241-253.

15. E. M. Stein, Boundary behavior of holomorphic functions of several complex variables, Princeton Univ. Press, Princeton, NJ, 1972.

16. R. Wallsten, Hankel operators between weighted Bergman spaces in the ball, Ark. Mat. 28 (1990), 183-192.

17. Kehe Zhu, Schatten class Hankel operators on the Bergman space of the unit ball, Amer. J. Math. 113 (1991), 147-168.

18. D. H. Phong and E. M. Stein, Estimates for the Bergman and Szegö projections on strongly pseudoconvex domains, Duke Math. J. 44 (1977), 695-704.

Department of Mathematics, SUNY at Buffalo, Buffalo, New York 14214

E-mail address: V087R5YM@UBVMS.CC.BUFFALO.EDU 